\title{
ACOUSTIC MODELING OF SHELL-ENCAPSULATED GAS BUBBLES
}

\author{
Peter J. A. FrinKING* and NICO DE JONG* ${ }^{*}$ \\ *Department of Experimental Echocardiography, Thoraxcenter, Erasmus University, Rotterdam, The Netherlands; and \\ ${ }^{\dagger}$ The Interuniversity Cardiology Institute of the Netherlands, Utrecht, The Netherlands
}

(Received 17 September 1997; in final form 19 January 1998)

\begin{abstract}
Existing theoretical models do not adequately describe the scatter and attenuation properties of the ultrasound contrast agents Quantison ${ }^{\mathrm{TM}}$ and Myomap ${ }^{\mathrm{TM}}$. An adapted version of the Rayleigh-Plesset equation, in which the shell is described by a viscoelastic solid, is proposed and validated for these agents and Albunex ${ }^{\circledR}$. The acoustic transmission and scattering are measured in the frequency band from 1-10 MHz. The measured transmission is used to estimate two parameters, the effective bulk modulus, $K_{e f f}$, describing the elasticity, and the friction parameter, $S_{F}$, describing the viscosity of the shell. For the scattering, the difference between measurements and calculations is $<3 \mathbf{d B}$. For Quantison ${ }^{\mathrm{TM}}$, the effective bulk modulus is independent of the bubble diameter. For Albunex ${ }^{\circledR}$, it increases for decreasing bubble diameter. The nonlinear response of Quantison ${ }^{\mathrm{TM}}$ is minimal for acoustic pressures up to $200 \mathrm{kPa}$. For acoustic pressures above $200 \mathrm{kPa}$, the measured scattering abruptly increases. This increase reaches a level of $20 \mathrm{~dB}$ for an acoustic pressure of $1.8 \mathrm{MPa}$. This response cannot be predicted by the theoretical model developed in this article. (c) 1998 World Federation for Ultrasound in Medicine \& Biology.
\end{abstract}

Key Words: Ultrasound, Contrast agent, Modeling.

\section{INTRODUCTION}

Nowadays conventional diagnostic ultrasound systems provide excellent information of structures and function of different kinds of organs. However, low volume flow or very small vessels associated with tumor vascularity often are difficult to detect using current diagnostic ultrasound systems. Furthermore, evaluating the perfusion of tissue to a certain organ currently is not feasible and may, in the future, become possible with the help of ultrasound contrast agents. Crucial criteria for ultrasound contrast agents to be successful include their ability to pass through the lung circulation after an intravenous injection and that they survive the maximum pressure developed in the left ventricle. For passing through the pulmonary system, the diameter should be less than around 8-10 $\mu \mathrm{m}$ (Hogg 1987). Free air bubbles (firstgeneration ultrasound contrast agents) of this size tend to disappear within $1 \mathrm{~s}$ (Epstein and Plesset 1950), depending on the dissolved gas concentration of the liquid. Encapsulating the small gas bubbles by a relatively thin

Address correspondence to: Peter J. A. Frinking, Experimental Echocardiography, Thoraxcentre, Erasmus University Rotterdam, P.O. Box 1738, 3000 DR Rotterdam, The Netherlands. E-mail: frinking@tch.fgg.eur.nl albumin shell (Albunex ${ }^{\circledR}$, Molecular Biosystems, San Diego, CA, USA) or stabilization by galactose (Levovist $^{\circledR}$, Schering AG, Berlin, Germany) prevents them from rapid disappearance. These are the so-called second-generation agents, which show good opacification of the left ventricular cavity. The third-generation contrast agents can be distinguished by prolongation of the lifetimes either by increasing the stiffness of the bubble shell (Quantison ${ }^{\mathrm{TM}}$, Andaris Ltd., Nottingham, UK; Sonovist $^{\circledR}$, Schering AG) or by using gases that dissolve poorly in blood (Optison ${ }^{\mathrm{TM}}$, Molecular Biosystems; Sonovue ${ }^{\mathrm{TM}}$, Bracco Research SA, Geneva, Switzerland; Imagent US, Alliance Pharmaceuticals, San Diego, CA, USA; EchoGen ${ }^{\circledR}$, Sonus Pharmaceuticals, Bothell, WA, USA; Aerosomes ${ }^{\circledR}$, ImaRx Pharmaceuticals, Tucson, AZ, USA). Thus, the microbubbles persist for a long time, allowing extensive examinations of heart functions, as well as the micro- and macrovasculatures. Using new technologies such as harmonic imaging (Burns et al. 1992), these agents allow echographic myocardial opacification.

\section{Previous related work}

Although bubble dynamics has been a field of research since the mid-1800s, a first theoretical description 
of the behavior of bubbles exposed to an external pressure field was developed by Lord Rayleigh (1917). Since then, much research has been carried out and resulted in an extensive description of the behavior of small gas bubbles under acoustic stimulation. Free gas bubbles are perfect scattering objects for ultrasound (Anderson and Hampton 1980; Medwin 1977; Miller 1981). However, encapsulated gas bubbles behave differently. Changed mechanical properties such as resonance and viscous or friction damping are introduced by the shell and influence the scattering properties.

de Jong et al. (1992, 1994a, 1994b) and de Jong and Hoff (1993) developed a theoretical description for the behavior of encapsulated gas bubbles, which was validated by extensive experimental results. Treating the shell as surface layers of elastic solids, they adapted Medwin's (1977) approach, describing the behavior of resonating gas bubbles and the Rayleigh-Plesset equation describing the nonlinear motion of encapsulated gas bubbles. By introducing elasticity and shell friction parameters, they calculated the scatter and extinction cross-section of Albunex ${ }^{\circledR}$. By fitting their model to transmission measurements, they were able to estimate these two parameters. They concluded that encapsulation results in an increase of the resonance frequency and a decrease of the scattering amplitude. They also found that the second-harmonic response for Albunex ${ }^{\circledR}$ is $20-30 \mathrm{~dB}$ lower than the fundamental response for an acoustic pressure of $50 \mathrm{kPa}$ and is much lower than for free gas bubbles.

Work done by Church (1995) is based on theoretical derivations and describes the effect of encapsulation on individual and collective bubble oscillations. By including the shell as a continuous layer of incompressible solid elastic material, he derived a Rayleigh-Plesset-like equation. The equation predicts that the surface layer supports a strain that counters the Laplace pressure and thereby stabilizes the bubble against dissolution. An analytical solution of this equation is presented, which includes both the fundamental and second-harmonic response. Adapting the dispersion relation, he showed that the change in acoustic velocity in liquids containing suspensions of bubbles is affected by the shell. All examples include the influence of the shell parameter from no shell to a shell elasticity of $150 \mathrm{MPa}$. For values of 88 $\mathrm{MPa}$ for the shell elasticity and $1.77 \mathrm{~Pa} \cdot \mathrm{s}$ for the shell viscosity, his conclusions agree with those of de Jong and Hoff (1993) concerning scattering and attenuation of Albunex ${ }^{\circledR}$.

Hoff (1996) proposed an approach describing the shell of the encapsulated bubble by a complex Young's modulus derived from the Kelvin-Voigt model for viscoelastic solids. The stiffness of the shell reduces the compressibility of the particles, resulting in an increase of the resonance frequency. The shell viscosity increases the damping constant of the particle-liquid system, decreasing the scattering amplitude. Implementing this in the linear model of Medwin (1977), the calculated attenuation spectra were fitted to the measured spectra, giving values for the shell elasticity and viscosity. He concludes that, with this model, the acoustic attenuation spectra can be predicted.

In this article, the ultrasound contrast agent Quantison $^{\mathrm{TM}}$ is described. First, an overview is given of the production process and basic properties, such as size and mechanical stability, are mentioned. Next, a RayleighPlesset-like equation will be derived describing the scatter and attenuation characteristics as a function of frequency of encapsulated gas bubbles. Experimental results show that theory and model agree reasonably well for Quantison ${ }^{\mathrm{TM}}$ as well as for other agents such as Myomap $^{\mathrm{TM}}$ (Andaris Ltd.), formerly known as Quantison Depot $^{\mathrm{TM}}$, and Albunex ${ }^{\circledR}$, which both consist of air bubbles encapsulated by an albumin shell. Besides the scatter and attenuation properties, the scattering-to-attenuation ratio (STAR), a measure for the scatter effectiveness of the contrast agent, will be discussed. Finally, the second-harmonic response of Quantison ${ }^{\mathrm{TM}}$ will be calculated. The approach described in this article is comparable with the viscoelastic approach described by Hoff (1996). However, his description is only valid for low acoustic amplitudes, i.e., in the linear range. In this article, a more general formula is developed, which describes both the linear and the nonlinear motion of the bubble.

\section{Quantison $^{\mathrm{TM}}$}

Quantison ${ }^{\mathrm{TM}}$ consists of air bubbles encapsulated by a shell of human albumin, which closely resembles native soluble human serum albumin. The shell is formed by spray-drying a solution of human albumin and is stabilized by heat fixation and chemical cross-linking. The final product is a dry powder that must be resuspended before use. The mean diameter is $3.2 \mu \mathrm{m}$, and less than $0.5 \%$ of the bubbles are $>6 \mu \mathrm{m}$. The size distribution is shown in Fig. 1. The shell thickness is 200-300 $\mathrm{nm}$ and proportional to the bubble diameter. Figure 2 shows an electron microscopic cross-section of a single fractionated Quantison ${ }^{\mathrm{TM}}$ bubble; from this figure the shell thickness can be appreciated. As a result of the relatively thick shell, the Quantison ${ }^{\mathrm{TM}}$ bubble is robust and able to withstand static pressures up to 600 $\mathrm{mmHg}$ without losing the air it contains. The density of the air-filled bubbles is approximately $780 \mathrm{~kg} / \mathrm{m}^{3}$. The particle density is about $1.5 \times 10^{9}$ bubbles per $\mathrm{mL}$, which corresponds to $8 \mu \mathrm{L}$ of air. 


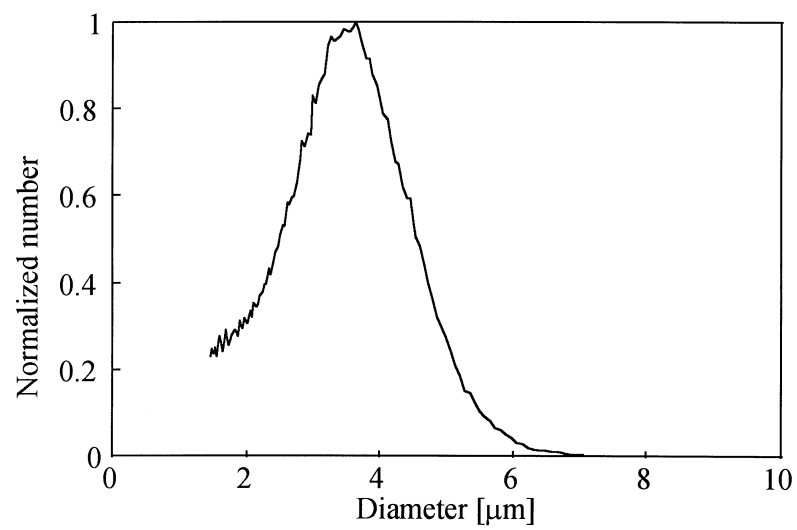

Fig. 1. Normalized size distribution of Quantison ${ }^{\mathrm{TM}}$ with diameters ranging from $1.46-8.3 \mu \mathrm{m}$. Distribution was measured with the Coulter Counter ${ }^{\circledR}$ Multisizer II with an aperture of 70 $\mu \mathrm{m}$ using 256 channels.

\section{THEORY}

To examine the dynamics of shell-encapsulated gas bubbles, a model developed by Rayleigh is used as the theoretical basis. The bubble is considered to be spherical and surrounded by an incompressible liquid of infinite extent. The liquid is assumed to be Newtonian, so the viscosity is constant. The bubble volume is defined by a single variable, the radius, and the motion is assumed to be spherically symmetrical. The wavelength of the ultrasound field is much larger than the radius of the bubble and only the motion of the bubble surface is of interest. The equation of motion of the bubble wall is given by (Leighton 1994):

$$
\rho R \ddot{R}+\frac{3}{2} \rho \dot{R}^{2}=p_{L}-p_{\infty},
$$

where $R$ is the instantaneous bubble radius, $\rho$ is the density of the surrounding liquid, $p_{L}$ is the liquid pressure at the bubble wall and $p_{\infty}$ is the liquid pressure at infinity. The liquid pressure at infinity can be expanded to $p_{o}+P(t)$, where $p_{o}$ is the hydrostatic pressure and $P(t)$ is the time-varying applied acoustic pressure. $\dot{R}$ and $\ddot{R}$ are the first and second derivatives of the radius with respect to time, respectively.

From this point, the present derivation deviates from the standard derivation of the Rayleigh-Plesset equation. The assumption is made that the presence of the shell completely dominates the motion of the bubble wall. Therefore, the bubbles are considered to have an effective bulk modulus, $K_{e f f}$, describing the elasticity, and a friction parameter, $S_{F}$, describing the viscosity of the shell. For a spherical volume $V$ deformed by a quasistatic pressure change $\Delta P$, which is uniform over the bubble surface, the volume strain is $-\Delta V / V$ and the effective bulk modulus is given by (Tabor 1987):

$$
K_{e f f}=-V \frac{\Delta P}{\Delta V}
$$

Since the volume is spherically symmetrical and defined by the radius, the volume strain can be written as:

$$
\frac{\Delta V}{V}=\left(\frac{R}{R_{o}}\right)^{3}-1,
$$

where $R_{o}$ is the equilibrium bubble radius. Combining eqns (2) and (3) gives:

$$
\Delta P=-K_{e f f}\left(\left(\frac{R}{R_{o}}\right)^{3}-1\right) .
$$

The pressure change $\Delta P$, which causes the volume change of the bubble, can be split into three parts: 1) the liquid pressure at the bubble wall $\left.\left(p_{L}\right), 2\right)$ the pressure caused by damping of the bubble-liquid system $\left(p_{d}\right)$ and 3 ) the hydrostatic pressure $\left(p_{o}\right)$, giving:

$$
\Delta P=p_{L}+p_{d}-p_{o} .
$$

Substitution of eqns (4) and (5) into eqn (1) and using the expanded expression for $p_{\infty}$ yields:

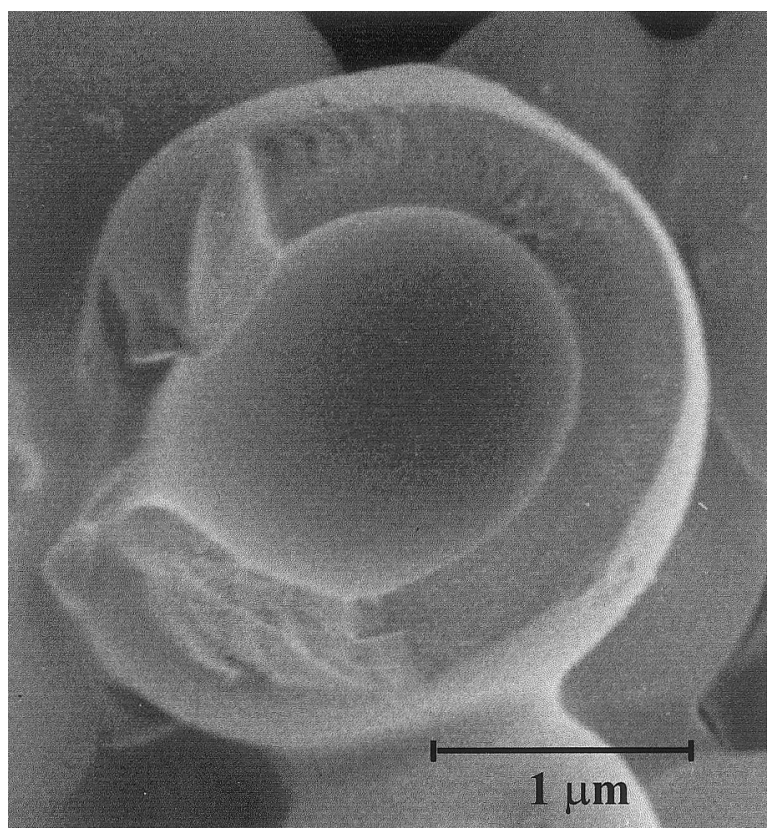

Fig. 2. Electron microscopic view of a "fractionated" Quantison $^{\mathrm{TM}}$ microsphere. 


$$
\rho R \ddot{R}+\frac{3}{2} \rho \dot{R}^{2}=-K_{\text {eff }}\left(\left(\frac{R}{R_{o}}\right)^{3}-1\right)-p_{d}-P(t) .
$$

An expression for $p_{d}$ can be derived from the equation of motion of the damped forced oscillator (Leighton 1994), equating the damping pressure, multiplied by the bubble surface, to the damping force:

$$
4 \pi R^{2} p_{d}=\beta \dot{R},
$$

where $\beta$ is the mechanical resistance. Together with the expressions of Medwin (1977) for the total damping coefficient, $\delta_{t o t}=\beta /(\omega m)$ and the effective mass $m=$ $4 \pi R^{3} \rho$, the damping pressure can be written as:

$$
p_{d}=\delta_{t o t} \rho \omega R \dot{R},
$$

where $\omega$ is the angular frequency. Equations (6) and (8) give the final expression:

$$
\begin{aligned}
\rho R \ddot{R}+ & \frac{3}{2} \rho \dot{R}^{2} \\
& =-K_{\text {eff }}\left(\left(\frac{R}{R_{o}}\right)^{3}-1\right)-\delta_{t o t} \omega \rho R \dot{R}-P(t),
\end{aligned}
$$

which is the Rayleigh-Plesset-like equation describing the motion of the wall of an encapsulated gas bubble.

The total damping coefficient $\delta_{\text {tot }}$ is the sum of four components:

$$
\delta_{t o t}=\delta_{r a d}+\delta_{v i s}+\delta_{t h}+\delta_{f r},
$$

where the first three terms on the right are described by Medwin (1977) viz $\delta_{r a d}$ is the damping due to reradiation, $\delta_{v i s}$ the damping due to shear viscosity in the surrounding medium and $\delta_{t h}$ the damping due to thermal conductivity. The last component, $\delta_{f r}$, is the damping due to internal friction or viscosity of the shell and is given by Hoff (1996):

$$
\delta_{f r}=\frac{3 S_{F}}{\omega \rho R^{2}}
$$

\section{Limitations}

Comparing eqn (9) with the standard RayleighPlesset equation for an ideal gas bubble (de Jong et al. 1994a), several comments can be made. The vapor pressure is neglected because, for a free air bubble in water at room temperature, its contribution is only around $1 \%$ of the bulk modulus. So, for encapsulated bubbles, which have a higher effective bulk modulus, its contribution will be even smaller. Surface tension is neglected be- cause there is no direct liquid-gas interface. The viscous and reradiation damping coefficients are assumed to be the same as for free gas bubbles, because they occur in the fluid and are independent of the presence of the shell. The presence of the shell has an effect on the thermal damping. It is considered that the shell has a heat capacity that is insufficient to exchange the energy from the gas to the liquid during expansion and compression. This means that there is no substantial net flow of heat into the liquid and that the process is adiabatic. Additional limitations can occur for nonlinear bubble oscillations. The damping coefficients as well as the effective bulk modulus and friction parameter are derived from linear theory and therefore are independent of the applied acoustic pressure.

\section{Scattering cross-section}

The solution of eqn (9) results in an instantaneous radius, velocity and acceleration of the bubble wall. The scattered sound pressure, $p_{s}(n \omega, R)$, at the bubble wall, including higher harmonics, in the frequency domain is (Coakley and Nyborg 1978):

$$
\left|p_{s}(n \omega)\right|=\left|\rho n \omega R_{o} \dot{R}(n \omega)\right|,
$$

where $n$ is the harmonic number $\left(1,2,3, \ldots, \frac{1}{2}, \frac{1}{3}, \ldots\right)$. The scattering cross-section, $\sigma_{s}(\omega, R)$, is used as the parameter defining the acoustic behavior of the bubble and is defined as the quotient of the scattered power and the incident acoustic intensity, $P(\omega)$. The general expression for the scattering cross-section in the frequency domain including higher harmonics is:

$$
\sigma_{s}(n \omega)=4 \pi R_{o}^{2} \frac{\left|p_{s}(n \omega)\right|^{2}}{|P(\omega)|^{2}} .
$$

\section{Mixture of bubbles}

For a mixture of bubbles, the scattering cross-section can be calculated under the assumption that: 1) the bubbles are uniformly and homogeneously distributed, and 2) multiple scattering is negligible. Thus, every bubble can be considered as an individual scattering object. For a given size distribution, the scattering crosssection of each individual bubble is multiplied by the respective number of each bubble size. The scattering coefficient (total scattering cross-section per unit volume), $\mu_{s}(\omega)$, then is obtained by summation of all the individual contributions according to $(n=1)$ :

$$
\mu_{s}(\omega)=\sum_{R} N(R) \sigma_{s}(\omega, R),
$$

where $N(R)$ is the bubble concentration. 
The total energy loss or attenuation for an acoustic beam traveling through a screen of bubbles is called the extinction coefficient, $\mu_{e}(\omega)$, and is given by (de Jong et al. 1992):

$$
\mu_{e}(\omega)=\mu_{a}(\omega)+\mu_{s}(\omega),
$$

where $\mu_{a}(\omega)$ is the absorption coefficient. Using the expression for the absorption coefficient derived by Coakley and Nyborg (1978):

$$
\mu_{a}(\omega)=\sum_{R} N(R) \sigma_{s}(\omega, R)\left(\frac{\delta_{t o t}(\omega, R)}{\delta_{r a d}(\omega, R)}-1\right),
$$

the extinction coefficient is given by:

$$
\mu_{e}(\omega)=\sum_{R} N(R) \sigma_{s}(\omega, R) \frac{\delta_{t o t}(\omega, R)}{\delta_{r a d}(\omega, R)}
$$

Values for the effective bulk modulus, $K_{\text {eff }}$, and the friction parameter of the bubble material, $S_{F}$ in eqn (9), are calculated by fitting the theoretically calculated extinction coefficient to the measured extinction coefficient by minimizing the absolute difference.

\section{Scatter-to-attenuation ratio}

The attenuation of the acoustic beam traveling through a screen of bubbles can cause shadowing of underlying biological structures and currently is not considered to be a useful parameter. An effective contrast agent therefore is defined by good scattering properties and low attenuation. In their work on standardization of ultrasound contrast agents, Bouakaz et al. (1998) suggest using, in addition to the backscatter coefficient, the scattering-to-attenuation ratio (STAR), as an improved measure for the effectiveness of the contrast agent. The STAR is defined as:

$$
\operatorname{STAR}(\omega)=\frac{\mu_{s}(\omega)}{\mu_{e}(\omega)} .
$$

Substituting eqn (15) into eqn (18) gives:

$$
\operatorname{STAR}(\omega)=\frac{\mu_{s}(\omega)}{\mu_{a}(\omega)+\mu_{s}(\omega)},
$$

where $\mu_{s}(\omega)$ represents that part of the energy that is scattered away omnidirectionally by the bubbles. On the other hand, $\mu_{a}(\omega)$ represents that part of the energy that is dissipated by the bubbles. Therefore, the lower the absorption of the incoming plane wave, the higher the
STAR. A maximum value of STAR $=1$ is obtained when there is no absorption.

\section{SIMULATION}

Equation (9) is solved by using the fourth-order Runga-Kutta method implemented using Matlab Simulink running on a Pentium 100 computer. A fixed stepsize was used to simplify fast Fourier transformations (FFT), which is related to the frequency of the applied acoustic wave. A sinusoidal wave was chosen and tapered taking a cosine window for the first five periods to quickly reach a steady-state condition. In this study, an acoustic pressure of $25 \mathrm{kPa}$ is used to calculate the linear response, and an acoustic pressure of $100 \mathrm{kPa}$ is used to calculate the nonlinear response. The initial values at $t=$ 0 are $R=R_{o}$ and $\dot{R}=0$. FFT is applied on the bubble wall velocity after steady state is reached. The remainder of the physical constants used are the liquid density, $\rho=$ $998 \mathrm{~kg} / \mathrm{m}^{3}$, and the liquid viscosity, $\eta=0.001 \mathrm{~Pa} \cdot \mathrm{s}$. All material properties are determined by their initial conditions and are considered to be constant during bubble oscillation, which is the lumped constant approach.

\section{MEASUREMENTS}

\section{Experimental set-up}

de Jong and Hoff (1993) described the measurement set-up that is used in this study. Three broadband (100\% of the central frequency at the $-20 \mathrm{~dB}$ level) singleelement transducers, with center frequencies of 2, 5 and $10 \mathrm{MHz}$ (Panametrics, Waltham, MA, USA), cover a total frequency band extending from 1-12 MHz. They all are focused at $75 \mathrm{~mm}$ and have apertures of 25, 18 and 12 $\mathrm{mm}$, respectively. The transducers are mounted in a water bath as illustrated in Fig. 3. Short, single-cycle, pulses are generated and received by a pulser/receiver (5052 PR, Panametrics). The received signals can be amplified from +40 to $-40 \mathrm{~dB}$ in steps of $2 \mathrm{~dB}$. The amplified signals are low-pass filtered to minimize noise and avoid aliasing and are digitized by a Lecroy 9400A (Lecroy, Chestnut Ridge, NY, USA) digital oscilloscope (100 MHz, 8 bits). A pulse generator (PM 5712, Philips, Stockholm, Sweden) is used for synchronization. The signals are recorded over a time window of $10 \mu \mathrm{s}$, with a sampling frequency of $50 \mathrm{MHz}$, and transferred to a personal computer (Compaq 386/20e) for further analysis.

The bubble container is made of PMMA (Perspex). The distance from the front to the back wall of the container is $60 \mathrm{~mm}$. The front has an angle of $15^{\circ}$ with the acoustic axis of the transducers, to minimize multiple reflections. At the level of the acoustic axis, an acoustic window with a diameter of $40 \mathrm{~mm}$ was made of $30-\mu \mathrm{m}$ thick TPX ${ }^{\circledR}$ foil (Mitsui Petrochemical Industries, Ltd., 


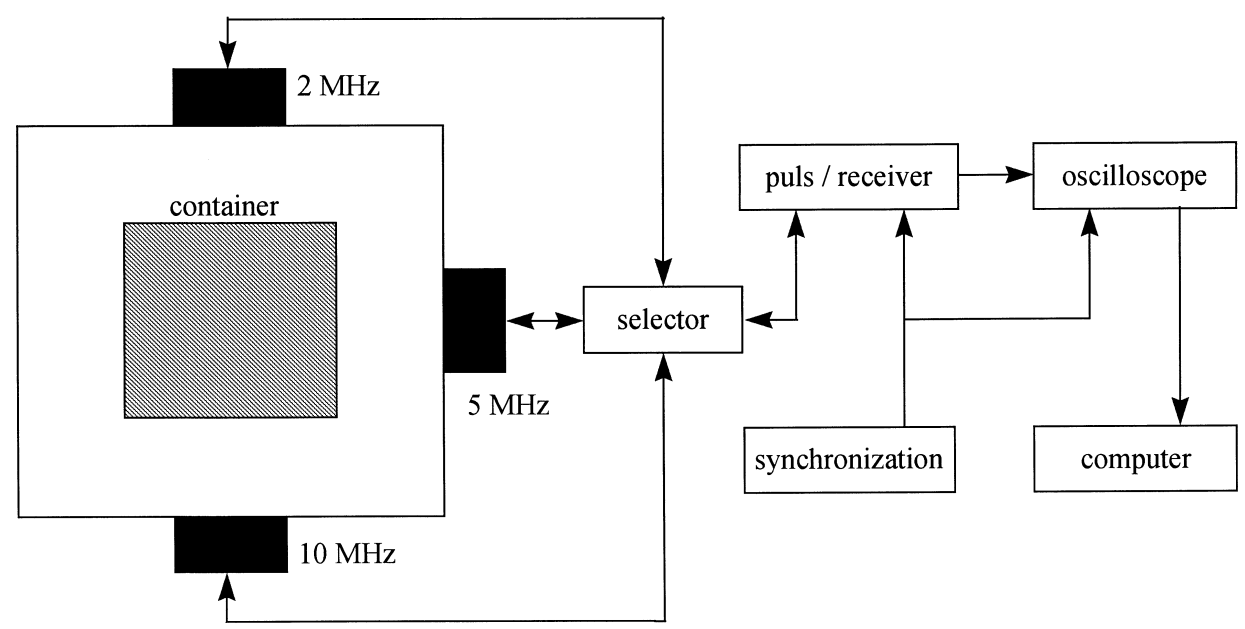

Fig. 3. Schematic set-up for acoustic scatter and attenuation measurements.

Tokyo, Japan). The back wall of the container is used as a flat plate reflector for reference measurements.

\section{Procedure}

The response of the back wall of the container, filled with pure Isoton ${ }^{\circledR}$ II (Coulter Electronics, Lutton, UK), placed at the focal distance of the transducers, is measured and used as the reference measurement, $I_{\text {ref }}(\omega)$. Then the contrast agent is added into the container, and the response of the back wall is measured again, $I_{\text {atten }}(\omega)$. The relationship between the measured and the calculated attenuation is given by (de Jong et al. 1992):

$$
\frac{I_{\text {atten }}(\omega)}{I_{r e f}(\omega)}=\exp \left(-\mu_{e}(\omega) d_{1}\right)
$$

where $d_{1}$ is the distance traveled through the bubble container.

To determine the scattering, first the container is repositioned with the front of the container at a distance of approximately $70 \mathrm{~mm}$ from the transducers. Then the intensity of the acoustic field scattered by the bubbles, $I_{\text {scatter }}(\omega)$, is measured. This measurement is normalized by the reference measurement, corrected for the perspex reflector. The relationship between the measured and the calculated scattering is given by (de Jong and Hoff 1993):

$$
\frac{I_{\text {scatter }}(\omega)}{I_{\text {ref }}(\omega)} \frac{16 z^{2}}{D^{2}}=\mu_{s}(\omega) d_{2}
$$

where $d_{2}$ is the window length, $z$ is the distance from the scattering volume to the transducer and $D$ is the diameter of the transducer. Equation (21) includes the correction for the limited aperture of the transducer and is only valid for curved transducers, placing the scattering volume in the focus where the far-field condition is valid. The scattered acoustic field is attenuated by the scatterers themselves as it travels through the suspension. The scattered signal $A(\omega)$ can be corrected for this attenuation by (de Jong and Hoff 1993):

$$
A_{\text {corr }}(\omega)=A(\omega) 10^{\frac{\alpha(\omega) d_{2}}{10}},
$$

where $A_{\text {corr }}(\omega)$ is the corrected scattered signal and $\alpha(\omega)$ is the attenuation at angular frequency $\omega$ (in $\mathrm{dB} / \mathrm{cm}$ ).

Every measurement consists of 62 traces to obtain an appropriate measurement of the complete distribution. After FFT, the average power spectra are calculated and smoothed using a moving window with a width of 200 $\mathrm{kHz}$ to remove radio frequency $(\mathrm{RF})$ noise. Before each measurement, the bubble suspension is stirred gently. The repetition rate was set to $1 \mathrm{~Hz}$ to ensure that the mixture changes enough to achieve independent scatter spectra from the recorded time traces.

\section{RESULTS}

The scatter and attenuation properties of samples of Quantison $^{\mathrm{TM}}$, Myomap ${ }^{\mathrm{TM}}$ and Albunex ${ }^{\circledR}$ were measured as described in the measurement section and calculated according to the model described in the theory section. All graphs show the results of eqns (20) and (21), expressed in $\mathrm{dB} / \mathrm{cm}$.

\section{Scattering and attenuation of Quantison ${ }^{\mathrm{TM}}$}

Figures 4A and 4B show the measured transmission (=1 - attenuation) and scattering properties of a 1:250 
A

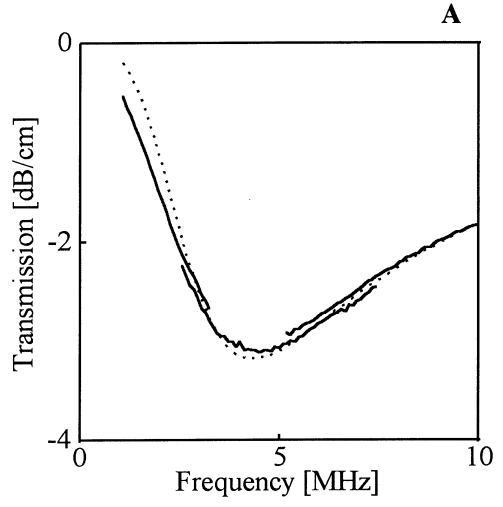

C

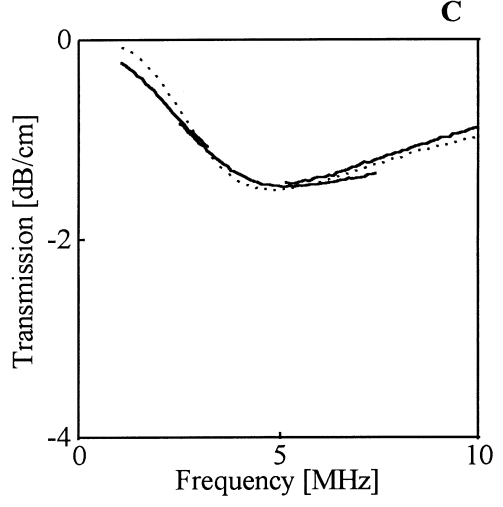

E

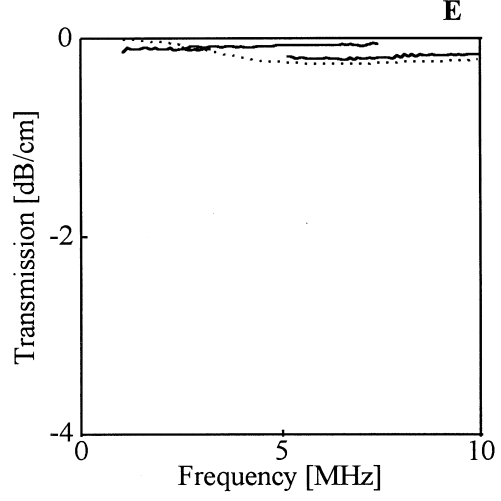

B

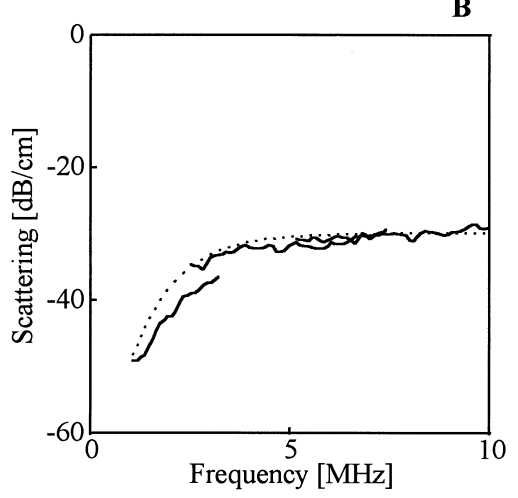

D
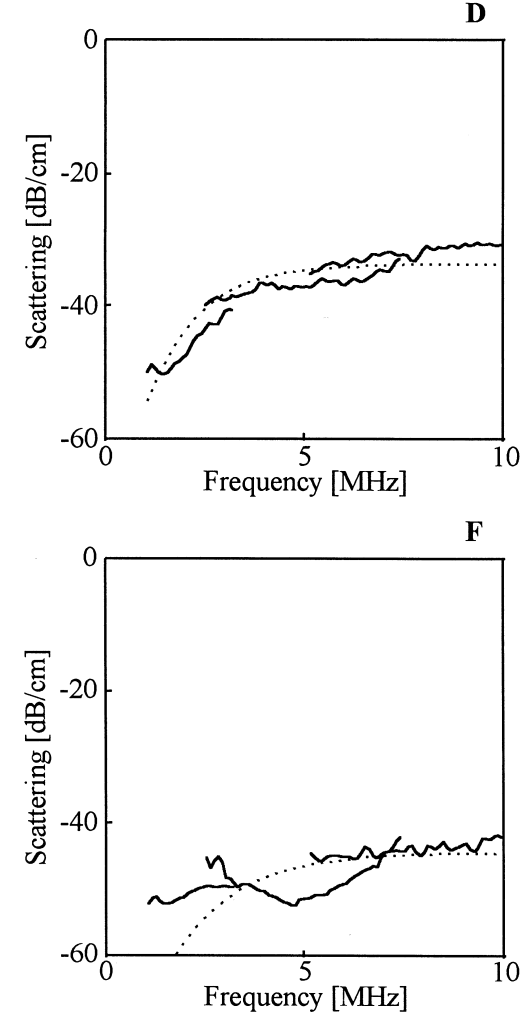

Fig. 4. Transmission and scatter vs. frequency for Quantison ${ }^{\mathrm{TM}} .-=$ measured spectrum; --- $=$ calculated spectrum using the size distribution shown in Fig. 1 . Bulk modulus $K_{\text {eff }}=17.4 \mathrm{MPa}$ and friction parameter $S_{F}=5.0 \mathrm{~Pa} \cdot \mathrm{s}$. (A,B) Unfiltered dilution of 1:250; (C,D) dilution of 1:150, filtered through a 5- $\mu \mathrm{m}$ filter; (E,F) dilution of 1:150, filtered through a $3-\mu \mathrm{m}$ filter. The variability in scattering is $<1 \mathrm{~dB}$ (not shown).

dilution of Quantison ${ }^{\mathrm{TM}}$, which corresponds to $6.6 \times 10^{6}$ microbubbles per $\mathrm{mL}$. The agreement at overlapping frequencies for the different transducers confirms the independence of the measurements on the transducer characteristics. A minimum in transmission at $4 \mathrm{MHz}$ of $-3 \mathrm{~dB} / \mathrm{cm}$ can be clearly appreciated and indicates the resonance frequency. At lower frequencies, a Rayleigh response can be observed, and above $4 \mathrm{MHz}$ the transmission increases with frequency, which is opposite to the behavior of normal biological tissue. The scattering of the same dilution is depicted in Fig. 4B and shows that the scattering is independent of the frequency above 4 $\mathrm{MHz}$, with a value of $-30 \mathrm{~dB} / \mathrm{cm}$. For lower frequencies, the scattering increases with frequency, again according to the Rayleigh theory.

After performing the acoustic measurements, the theoretical transmission spectrum (dotted line in Fig. 4A) is calculated by using the measured size distribution of the same Quantison ${ }^{\mathrm{TM}}$ batch (Fig. 1). Minimizing the absolute difference between these two spectra results in 

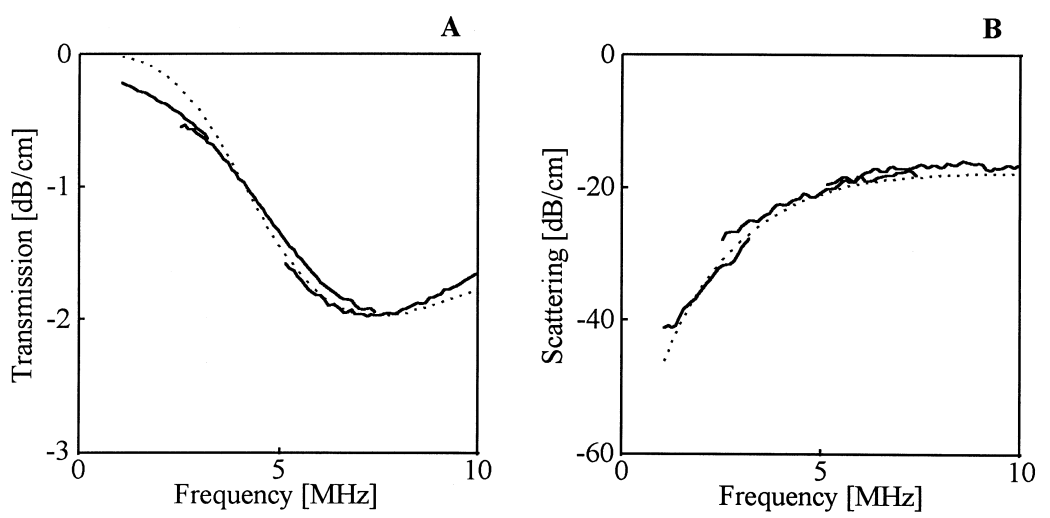

Fig. 5. (A) Transmission and (B) scatter vs. frequency for Myomap ${ }^{\mathrm{TM}} .-=$ measured spectrum;

$---=$ calculated spectrum using corresponding size distribution (Fig. 6.). $K_{\text {eff }}=78.4 \mathrm{MPa}$ and $S_{F}=5.1 \mathrm{~Pa} \cdot \mathrm{s}$.

values of $K_{\text {eff }}=17.4 \mathrm{MPa}$ and $S_{F}=5.0 \mathrm{~Pa} \cdot \mathrm{s}$. These parameters are then used, together with the size distribution, to calculate the scattering (dotted line in Fig. 4B).

Figures $4 \mathrm{C}-4 \mathrm{D}$ and $4 \mathrm{E}-4 \mathrm{~F}$ show the results for a filtered Quantison ${ }^{\mathrm{TM}}$ dilution of 1:150 $\left(11 \times 10^{6}\right.$ microbubbles per $\mathrm{mL}$ ) with Nuclepore ${ }^{\circledR}$ (Coster Corp., Cambridge, MA, USA) mechanical filters with pore sizes of 5 and $3 \mu \mathrm{m}$, respectively. Thus, the influence of different parts of the size distribution on the scatter and attenuation characteristics is determined. For the $5-\mu \mathrm{m}$ filtered dilution, a less dominant minimum in transmission can still be appreciated at a frequency of $5 \mathrm{MHz}$. For the $3-\mu \mathrm{m}$ filtered dilution, the transmission is practically flat. This indicates that the main contribution to attenuation is due to bubbles $>3 \mu \mathrm{m}$ in diameter. The results for the scattering show that there is a decrease in scatter level as function of bubble diameter. Also, there is an increase in frequency, from $4-6 \mathrm{MHz}$, above which the scattering is independent of the frequency. For both dilutions, the corresponding size distributions were measured with the Coulter Counter ${ }^{\circledR}$ (Coulter Electronics) Multisizer II and used to calculate the theoretical transmission and scattering curves. In both calculations, the values for the effective bulk modulus and friction parameter determined in Figs. 4A and 4B were used. This means that these parameters are independent of the bubble diameter and results from the fact that the shell thickness is proportional to the bubble diameter. In all experiments, the difference between the measured and calculated scattering is about $3 \mathrm{~dB}$. For the $3-\mu \mathrm{m}$ filtered dilution, it was difficult to measure the scattering below $5 \mathrm{MHz}$. Because of the low concentration of bubbles after filtering, the scattered signal hardly exceeded the noise level.

\section{Scattering and attenuation of Myomap ${ }^{\mathrm{TM}}$}

Figures $5 \mathrm{~A}$ and $5 \mathrm{~B}$ show the measured and calculated results for a Myomap ${ }^{\mathrm{TM}}$ dilution of 1:150 $\left(1 \times 10^{5}\right.$ microbubbles per $\mathrm{mL}$ ). This agent, which also consists of air bubbles encapsulated by a shell of human albumin such as Quantison ${ }^{\mathrm{TM}}$, has a mean size of $10 \mu \mathrm{m}$ (Fig. 6). The calculated values for the effective bulk modulus and friction parameter are $K_{\text {eff }}=78.4 \mathrm{MPa}$ and $S_{f}=5.1$ $\mathrm{Pa} \cdot \mathrm{s}$. The large bulk modulus of Myomap TM is a result of a higher stiffness compared to Quantison ${ }^{\mathrm{TM}}$. This is caused by the shell, which is more than three times thicker than the Quantison ${ }^{\mathrm{TM}}$ shell. This also explains why, although the bubbles are larger than the Quanti$\operatorname{son}^{\mathrm{TM}}$ bubbles (i.e., expecting a lower resonance frequency), the resonance frequency is higher instead.

\section{Scattering and attenuation of Albunex ${ }^{\circledR}$}

Figures 7A and 7B show the results for Albunex ${ }^{\circledR}$. The size distribution and results of previously published measurements (de Jong and Hoff 1993) of filtered Albunex ${ }^{\circledR}$ with a $12-\mu \mathrm{m}$ pore size mechanical filter were used

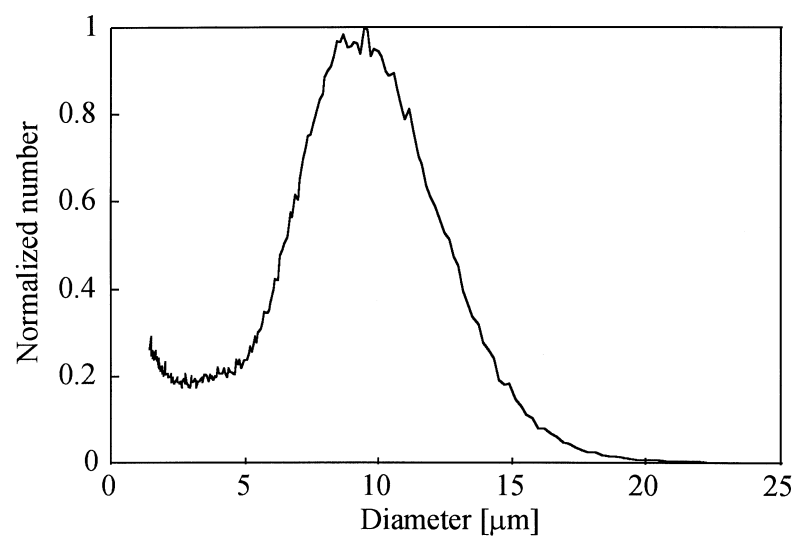

Fig. 6. Normalized size distribution of Myomap ${ }^{\mathrm{TM}}$ with diameters ranging from 1.46-22.9 $\mu \mathrm{m}$. Distributions was measured with the Coulter Counter ${ }^{\circledR}$ Multisizer II with an aperture of 70 $\mu \mathrm{m}$ using 256 channels. 
A

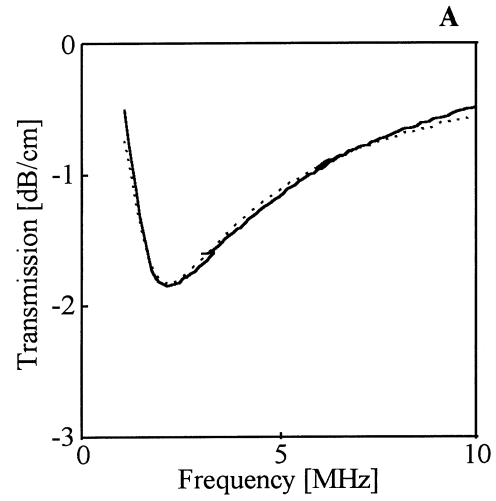

B

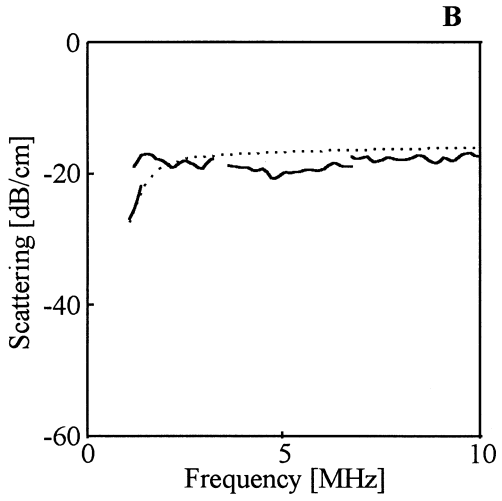

Fig. 7. (A) Transmission and (B) scatter vs. frequency for Albunex ${ }^{\circledR}$. $-=$ measured spectrum; ---- = calculated spectrum using corresponding size distribution. $K_{\text {eff }}=1.3 \mathrm{MPa}$ and $S_{F}=0.1 \mathrm{~Pa} \cdot \mathrm{s}$.

for the calculation of the effective bulk modulus and friction parameter. The calculated values are $K_{\text {eff }}=1.3$ $\mathrm{MPa}$ and $S_{f}=0.1 \mathrm{~Pa} \cdot \mathrm{s}$. Also, the measurements for Albunex ${ }^{\circledR}$ filtered with 8-, 5- and 3- $\mu \mathrm{m}$ mechanical filters (de Jong and Hoff 1993) were used, and the values for $K_{\text {eff }}$ and $S_{f}$ are, respectively: $2.0 \mathrm{MPa}$ and $0.1 \mathrm{~Pa} \cdot \mathrm{s}$, 6.2 $\mathrm{MPa}$ and $0.11 \mathrm{~Pa} \cdot \mathrm{s}$ and $9.8 \mathrm{MPa}$ and $0.11 \mathrm{~Pa} \cdot \mathrm{s}$. In this case, the effective bulk modulus and friction parameter are dependent on the bubble diameter. This confirms data reported by Hoff (1996) and is explained by the fact that the thickness of the Albunex ${ }^{\circledR}$ shell is independent of the diameter and, therefore, the stiffness increases for smaller bubbles.

\section{STAR of Quantison ${ }^{\mathrm{TM}}$ and Myomap ${ }^{\mathrm{TM}}$}

Using eqn (18), the STAR is calculated for Quantison $^{\mathrm{TM}}$ and Myomap ${ }^{\mathrm{TM}}$. Figure 8 depicts a bar graph of the STAR at five different frequencies. For Quantison ${ }^{\mathrm{TM}}$, the STAR is low due to a high stiffness and friction parameter of the shell and increases as function of frequency. For frequencies between 1 and $10 \mathrm{MHz}$, which are used for medical diagnosis, the STAR is around $0.1 \%$, meaning that the absorption is 1,000 times larger than the scattering. For Myomap $^{\text {TM }}$ the STAR is higher in spite of a higher stiffness. However, the bubbles are larger and the friction parameter is almost similar to the friction parameter of Quantison $^{\mathrm{TM}}$, giving a higher scattering and lower absorption compared to Quantison ${ }^{\mathrm{TM}}$. The value of the STAR is around $2-3 \%$ at $5 \mathrm{MHz}$, meaning that the absorption is 50 times larger than the scattering.

\section{Nonlinear behavior of Quantison ${ }^{\mathrm{TM}}$}

One of the special characteristics of gas bubbles is that the bubble starts to oscillate nonlinearly when the applied acoustic pressure is increased (de Jong et al. 1994a; Miller 1981; Schrope et al. 1992). This becomes extremely apparent for small bubbles. However, the non- linear response of encapsulated bubbles is much smaller. Figure 9 shows the result of eqn (9) for an applied acoustic pressure of $100 \mathrm{kPa}$, by using $n=1$ and $n=2$ in eqn (13), i.e., calculating the fundamental (Fig. 9A) and second-harmonic responses (Fig. 9B) of Quanti$\operatorname{son}^{\mathrm{TM}}$. A $60-\mathrm{dB}$ difference can be observed between the fundamental and second-harmonic response, and it is obvious from this result that Quantison ${ }^{\mathrm{TM}}$ shows negligible second-harmonic response at this acoustic pressure. The lack of harmonic response was confirmed experimentally for applied acoustic pressures up to $100 \mathrm{kPa}$. No harmonic components above the noise level $(-55$ $\mathrm{dB}$ ) could be measured.

\section{DISCUSSION AND CONCLUSIONS}

In this article, the idea introduced by Hoff (1996) to treat the shell of an encapsulated gas bubble as a viscoelastic solid was expanded to a Rayleigh-Plesset-like equation. The effective bulk modulus and friction param-

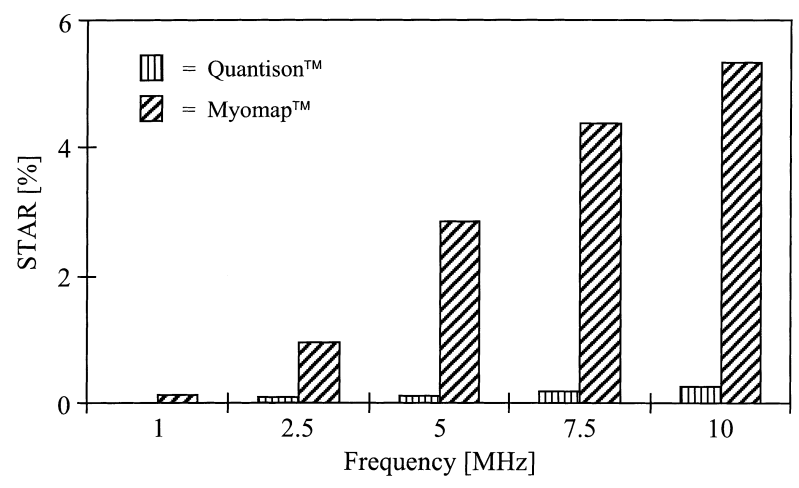

Fig. 8. The scattering-to-attenuation ratio (STAR) for Quantison $^{\mathrm{TM}}$ and Myomap ${ }^{\mathrm{TM}}$ at frequencies of 1, 2.5, 5, 7.5 and 10 $\mathrm{MHz}$. The variability is around $1 \%$ of the values (not shown). 

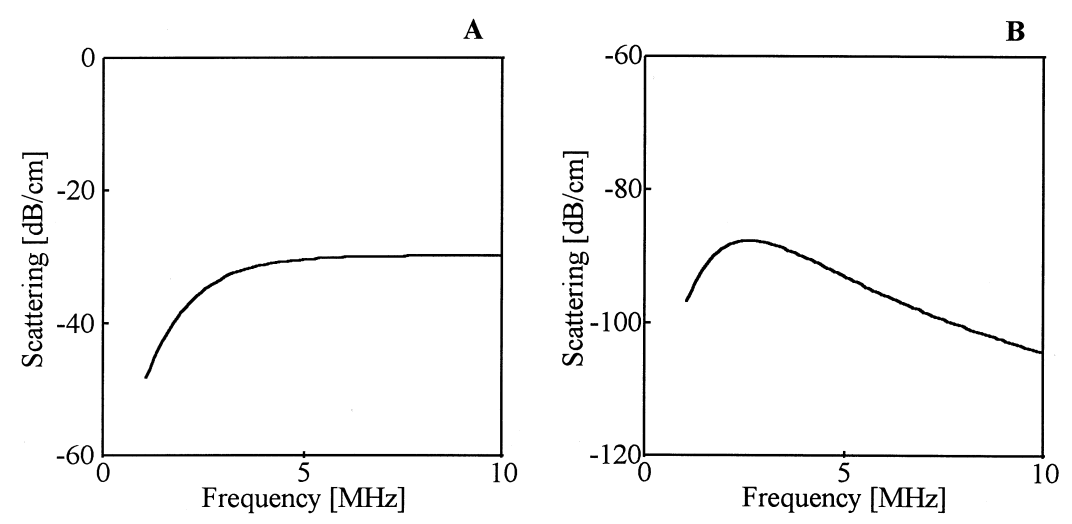

Fig. 9. Calculated scatter spectra of Quantison ${ }^{\mathrm{TM}}$ using the size distribution shown in Fig. 1. Bulk modulus $K_{\text {eff }}=17.4$ $\mathrm{MPa}$ and friction parameter $S_{F}=5.0 \mathrm{~Pa} \cdot \mathrm{s}$. Left panel shows fundamental response $[n=1$ in eqn (13)]; right panel shows second-harmonic response $[n=2$ in eqn (13)] at an acoustic pressure of $100 \mathrm{kPa}$.

eter, describing the elasticity and viscosity of the shell, are found by fitting the calculated transmission spectrum to the measured transmission spectrum. The same parameters then are used to calculate the scattering. This model was used to calculate the transmission and scattering of the ultrasound contrast agents Quantison ${ }^{\mathrm{TM}}$, Myomap $^{\mathrm{TM}}$ and Albunex ${ }^{\circledR}$. For all the results, the difference between measured and calculated scattering is $<3$ $\mathrm{dB}$.

The effective bulk modulus of Quantison ${ }^{\mathrm{TM}}$ (17.4 $\mathrm{MPa}$ ) is 124 times the bulk modulus of air, which at adiabatic conditions and an ambient pressure of $1 \mathrm{~atm}$ is $0.14 \mathrm{MPa}$. However, compared to water $(2,250 \mathrm{MPa})$, this is only $0.7 \%$. For Albunex ${ }^{\circledR}$, the effective bulk modulus (1.3 MPa) is nine times the bulk modulus of air. This demonstrates the dominant character of the shell, even for an encapsulated gas bubble with a shell thickness of $15 \mathrm{~nm}$ (Church 1995).

For Quantison ${ }^{\mathrm{TM}}$, the shell thickness is proportional to the bubble diameter, meaning that the stiffness and, therefore, the effective bulk modulus, are independent of the bubble diameter. For Albunex ${ }^{\circledR}$, however, the shell thickness is independent of the bubble diameter (de Jong et al. 1992), i.e., the stiffness increases for smaller bubbles. This means that the effective bulk modulus is dependent on the bubble diameter and increases with decreasing bubble diameter. The dependence of the effective bulk modulus of Albunex ${ }^{\circledR}$ on the bubble diameter also is reported by Hoff (1996).

With previous models (de Jong and Hoff 1993; de Jong et al. 1992, 1994a; Church 1995) it is not possible to calculate the transmission and scattering of Quantison $^{\mathrm{TM}}$ and Myomap ${ }^{\mathrm{TM}}$. Both models are specifically developed for Albunex ${ }^{\circledR}$, i.e., encapsulated gas bubbles with a relatively thin shell. This approach does not hold if the shell occupies a considerable part of the bubble volume (e.g., Quantison ${ }^{\mathrm{TM}}$ and Myomap $\left.{ }^{\mathrm{TM}}\right)$. The fact that the transmission and scattering of Albunex ${ }^{\circledR}$ can be calculated with the present model, as well, indicates that this model is suited for thick and thin shells.

The assumptions made to neglect vapor pressure and surface tension should be reconsidered for modeling other agents. Different methods for stabilizing the gas can have an effect on these assumptions. Also, a substantial net flow of heat into the liquid may occur in these situations. This means that the process should be considered polytropic instead of adiabatic. For free gas bubbles, however, the model described in this article no longer holds and the standard Rayleigh-Plesset equation should be used.

The STAR can be used to describe the acoustic efficiency of ultrasound contrast agents. The STAR of Quantison ${ }^{\mathrm{TM}}$ and Myomap ${ }^{\mathrm{TM}}$ was calculated and ranges from $0.1 \%-5 \%$, respectively, for frequencies between 1 and $10 \mathrm{MHz}$. The STAR for Albunex ${ }^{\circledR}$ ranges from 2\%-18\% [Bouakaz et al. (1998)] in the same frequency range. This means that Albunex ${ }^{\circledR}$ shows a higher acoustic efficiency than Quantison ${ }^{\mathrm{TM}}$. However, these values are only valid for low acoustic pressures. At high acoustic pressures, nonlinear transient effects appear. It has been reported (de Jong et al. 1996; Frinking and de Jong 1997) that, above an acoustic pressure of $200 \mathrm{kPa}$, the measured scattering coefficient of Quantison ${ }^{\mathrm{TM}}$ abruptly increases. This increase reaches a level of $20 \mathrm{~dB}$ for an acoustic pressure of $1.8 \mathrm{MPa}$. This response cannot be predicted by the theoretical model developed in this article (Fig. 10). Measurement and calculation agree for applied pressures $<200 \mathrm{kPa}$. For applied pressures $>$ $200 \mathrm{kPa}$, the measurement and calculation deviate.

From Fig. 9, it is shown that Quantison ${ }^{\mathrm{TM}}$ shows hardly any second-harmonic response at an acoustic pressure of $100 \mathrm{kPa}$. Even for acoustic pressures up to 2 $\mathrm{MPa}$, the model developed in this article predicts a second-harmonic response that still does not exceed the 


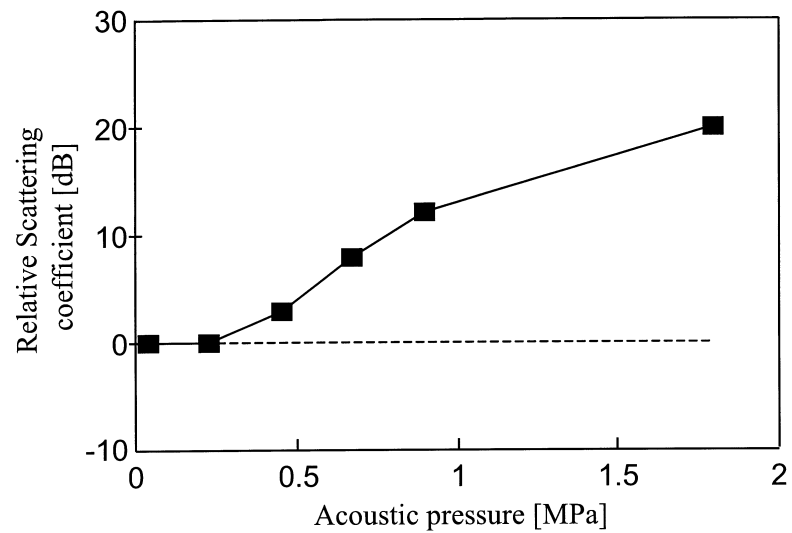

Fig. 10. Relative measured (- - ) and calculated (---) scattering coefficient of Quantison ${ }^{\mathrm{TM}}$ as a function of the applied acoustic pressure.

noise level of $-55 \mathrm{~dB}$. However, it has been reported (de Jong et al. 1996; Frinking and de Jong 1997) that, above an acoustic pressure of $200 \mathrm{kPa}$, the spectrum of the measured scattering signal broadens up and contains harmonics with amplitudes comparable to the amplitude of the fundamental frequency.

We have developed a theoretical model that describes accurately the linear and nonlinear response of encapsulated gas bubbles. However, the model does not describe the abrupt increase in scattering at applied acoustic pressures $>$ $200 \mathrm{kPa}$ (Fig. 10). An explanation is that, at these high acoustic pressures, free air bubbles are released from the Quantison $^{\mathrm{TM}}$ microspheres. This phenomenon is described in detail by Frinking and de Jong (1997).

Acknowledgement-The authors thank Dr. E. Ignacio Cespedes for reviewing the paper.

\section{REFERENCES}

Anderson AL, Hampton LD. Acoustics of gas-bearing sediments I. Background. J Acoust Soc Am 1980;67:1865-1889.
Bouakaz A, de Jong N, Cachard C. Standardization procedure for in-vitro acoustic characterization of ultrasound contrast agents. Ultrasound Med Biol 1998;24:469-472.

Burns PN, Powers JE, Fritzsch T. Harmonic imaging: A new imaging and Doppler method for contrast enhanced ultrasound. Radiology 1992;185(P): 142

Church C. The effects of an elastic solid surface layer on the radial pulsations of gas bubbles. J Acoust Soc Am 1995;97:1510-1521.

Coakley WT, Nyborg LN. Cavitation: Dynamics of gas bubbles; Applications. In: Fry FJ, ed. Ultrasound: Its applications in medicine and biology. Amsterdam: Elsevier Scientific Publishing Company, 1978;3:77-159

de Jong N, Hoff L, Skotland T, Bom N. Absorption and scatter of gas filled microspheres: Theoretical considerations and some measurements. Ultrasonics 1992;30:95-103.

de Jong N, Hoff L. Ultrasound scattering of Albunex ${ }^{\circledR}$ microspheres. Ultrasonics 1993;31:175-181.

de Jong N, Cornet R, Lancée CT. Higher harmonics of vibrating gas filled microspheres. Part one: Simulations. Ultrasonics 1994a;32: 447-453.

de Jong N, Cornet R, Lancée CT. Higher harmonics of vibrating gas filled microspheres. Part two: Measurements. Ultrasonics 1994b; $32: 455-459$

de Jong N, Frinking PJA, Ten Cate FJ, van der Wouw P. Characteristics of contrast agents and 2D imaging. Proc IEEE Ultrason Symp 1996;2:1449-1458.

Epstein PS, Plesset MS. On the stability of gas bubbles in liquid-gas solutions. J Chem Phys 1950;18:1505-1509.

Frinking PJA, de Jong N. Modeling of ultrasound contrast agents. Proc IEEE Ultrason Symp 1997;2:1601-1604.

Hoff L. Acoustic properties of shell-encapsulated, gas-filled ultrasound contrast agents. Proc IEEE Ultrason Symp 1996;2:1441-1444.

Hogg JC. Neutrophil kinetics and lung injury. Physiol Rev 1987;67: 1249-1295.

Leighton TG. The acoustic bubble. London: Academic Press, 1994.

Medwin M. Counting bubbles acoustically: A review. Ultrasonics 1977:15:7-13.

Miller DL. Ultrasonic detection of resonant cavitation bubbles in a flow tube by their second-harmonic emission. Ultrasonics 1981;19:217224.

Rayleigh, Lord (Strutt JW). On the pressure developed in a liquid during the collapse of a spherical cavity. Philos Mag 1917;34:9498.

Schrope B, Newhouse VL, Uhlendorf V. Simulated capillary blood flow measurement using a nonlinear ultrasound contrast agent. Ultrason Imaging 1992;14:134-158.

Tabor D. Gasses, liquids and solids. New York: Cambridge University Press, 1987. 